\title{
Analysis of compaction initiation in human embryos by using time-lapse cinematography
}

\author{
Kyoko Iwata • Keitaro Yumoto • Minako Sugishima • \\ Chizuru Mizoguchi • Yoshiteru Kai • Yumiko Iba • \\ Yasuyuki Mio
}

Received: 1 November 2013 / Accepted: 10 February 2014 / Published online: 9 March 2014

(C) Springer Science+Business Media New York 2014

\begin{abstract}
Purpose To analyze the initiation of compaction in human embryos in vitro by using time-lapse cinematography (TLC), with the goal of determining the precise timing of compaction and clarifying the morphological changes underlying the compaction process.

Methods One hundred and fifteen embryos donated by couples with no further need for embryo-transfer were used in this study. Donated embryos were thawed and processed, and then their morphological behavior during the initiation of compaction was dynamically observed via time-lapse cinematography (TLC) for 5 days.

Results Although the initiation of compaction occurred throughout the period from the 4-cell to 16-cell stage, 99 ( $86.1 \%$ ) embryos initiated compaction at the 8-cell stage or later, with initiation at the 8-cell stage being most frequent (22.6\%). Of these 99 embryos, $49.5 \%$ developed into goodquality blastocysts. In contrast, of the 16 (13.9\%) embryos that initiated compaction prior to the 8 -cell stage, only $18.8 \%$ developed into good-quality blastocysts. Embryos that initiated compaction before the 8-cell stage showed significantly higher numbers of multinucleated blastomeres, due to asynchronism in nuclear division at the third mitotic division resulting from cytokinetic failure.
\end{abstract}

Capsule In the human embryos in vitro, compaction initiated at the third mitotic division or later, and multinucleated blastomeres (MNBs) were associated with cytoplasmic failure accompanied by karyokinesis, particularly when compaction occurred before the 8-cell stage.

Electronic supplementary material The online version of this article (doi:10.1007/s10815-014-0195-2) contains supplementary material, which is available to authorized users.

K. Iwata $(\bowtie) \cdot$ K. Yumoto $\cdot$ M. Sugishima $\cdot$ C. Mizoguchi $\cdot$ Y. Kai $\cdot$ Y. Iba $\cdot$ Y. Mio

Reproductive Centre, Mio Fertility Clinic, 2-1-1 Kuzumo-minami,

Yonago, Japan

e-mail: mfc-rep@mfc.or.jp
Conclusions The initiation of compaction primarily occurs at the third mitotic division or later in human embryos. Embryos that initiate compaction before the 8-cell stage are usually associated with aberrant embryonic development (i.e., cytokinetic failure accompanied by karyokinesis).

Keywords Time-lapse cinematography (TLC) · Compaction - Multinucleated blastomere (MNB) - Embryo · Cytokinetic failure

\section{Introduction}

The first morphologically evident event in the differentiation of mammalian embryos is the process of compaction, which in turn has an important influence on the subsequent processes of blastocyst formation, the initiation of inner cell mass formation, and trophectoderm differentiation. During compaction, unique cell surface changes occur, involving microvilli redistribution, changes to the gap and tight junctions, mitotic proliferation, and cytoplasmic polarization. Cell-to-cell adhesion increases and intercellular contacts are maximized until the boundaries between cells become obscured, with the resultant formation of the morula. The expression of these functions requires zygotic transcription and differentiation into distinct cell lineages, which occurs at the 2-cell stage in the mouse [1-3].

The onset of compaction varies among species. Compaction is most prominent in the mouse, where it appears at the 8-cell stage, whereas in bovines it begins at the 16-cell to 32-cell stage, and in the rabbit it commences at the 32-cell to 64-cell stage $[2,4,5]$. Compaction is less marked in the rhesus monkey and baboon, where it appears at the 16-cell to 32-cell stage, and in pig embryos compaction is not established until shortly before blastocyst formation [1, 6-8]. Moreover, microstructural analyses of the cell surfaces during compaction in 
primate embryos, including those of humans, revealed blastomeres that were flattened against each other, with a reduction of the intervening cleft, while the length and density of the microvilli gradually increased until obvious changes became apparent at day $4[2,9]$. However, little is known regarding the exact details of this critical event in human embryogenesis, and both the precise timing of compaction and the underlying mechanisms controlling the process have yet to be clarified [2].

Time-lapse cinematography (TLC) techniques, originally developed by the authors based on a study by Payne [10], have enabled us to precisely observe the morphology of human embryonic development in vitro. TLC provides highresolution, continuous imaging, in which the various components of the cell can be distinguished and monitored during the recording period. Thus far, we have used this technique to study the fertilization process and embryonic development up to hatching [11-13].

Here, we used TLC to analyze the initiation of compaction in human embryos in vitro. We observed several novel phenomena during the initiation of compaction that were closely related to the formation of multinucleated blastomeres (MNBs).

\section{Materials and methods}

TLC was conducted as described previously [11, 12]. Briefly, we used an inverted microscope (IX-71; Olympus, Tokyo, Japan) with Nomarski differential interference contrast (DIC) optics (Olympus) and a micromanipulator (Narishige, Japan) covered with a handcrafted chamber made of acrylic resin. An air heater was placed in the corner of the chamber to maintain the optimal temperature. Our system also contained a small acrylic chamber surrounded by a small water bath on the stage of the microscope. A glass-bottom dish with a

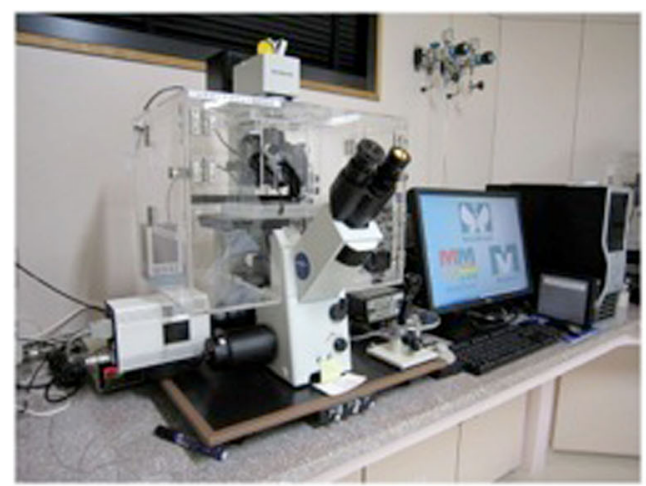

Fig. 1 Time-lapse cinematography (TLC). The inverted microscope was equipped with a CCD digital camera (Roper Scientific Photometrics, USA) connected to a computer, and images were displayed via MetaMorph (Universal Imaging Co., USA). Digital images of the microdrop of culture medium $(5 \mu \mathrm{L})$ was placed in the center of the small chamber. Humidified $\mathrm{CO}_{2}$ gas was infused into the chamber through the water bath, and the volume of flowing $\mathrm{CO}_{2}$ and temperature were adjusted to the optimal values (temperature, $37.0 \pm 0.2^{\circ} \mathrm{C} ; \mathrm{pH}, 7.37 \pm 0.05$ ) within the microdrop covered with mineral oil (Sage, Pasadena, CA). The inverted microscope was equipped with a charge-coupled device (CCD) digital camera (Roper Scientific Photometrics, Tucson, AZ), which was connected to a computer, and images were displayed by using MetaMorph software (Universal Imaging Co, Downingtown, PA). In total, 2,000 to 8,000 digital images of each cultured embryo were acquired over a period of 5 days, with an exposure time of 1/20 s. (Fig. 1).

One hundred and nineteen embryos were donated by couples with no further need for embryo transfer, and these were used in this study after obtaining informed consent. The donated embryos had been cryopreserved at an early stage (pronuclear stage to 4-cell stage) via the slow-cooling method (Air water Inc., Japan) or ultra-rapid vitrification (Kitazato Co., Japan). Cleavage stage embryos were graded by Veeck's criteria [14] and blastocyst stage embryo were graded as per Gardner's grading system [15]. After thawing, the embryos were cultured for 24 to $48 \mathrm{~h}$, and embryo quality was assessed via the modified Veeck's criteria. Blastocyst stage embryos that were evaluated as good quality (i.e., quality $3 \mathrm{BB}$ or greater according to the Gardner grading system) were used for time-lapse recording. Of the 119 embryos donated, 115 successfully developed to the blastocyst stage, and were included in the study.

\section{Ethical approval}

This study was approved by the ethics committee of the Japanese Institution for Standardizing Assisted Reproductive Technology (JISART).

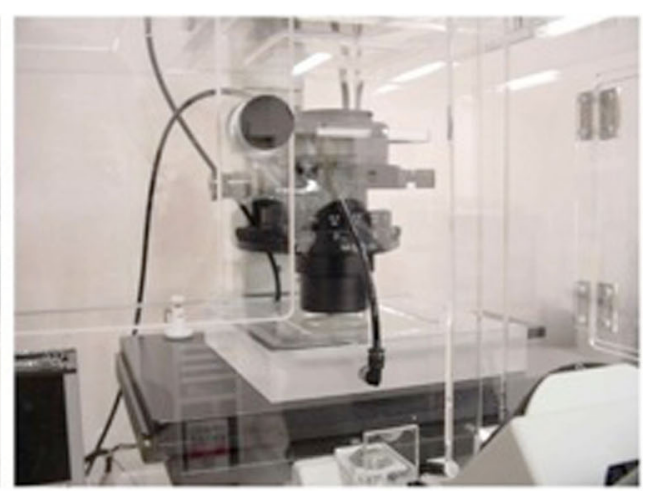

cultured embryos were acquired for approximately $40 \mathrm{~h}$, with an exposure time of $50 \mathrm{~ms}$. In total, 2,000 to 8,000 images were taken during the observation period 

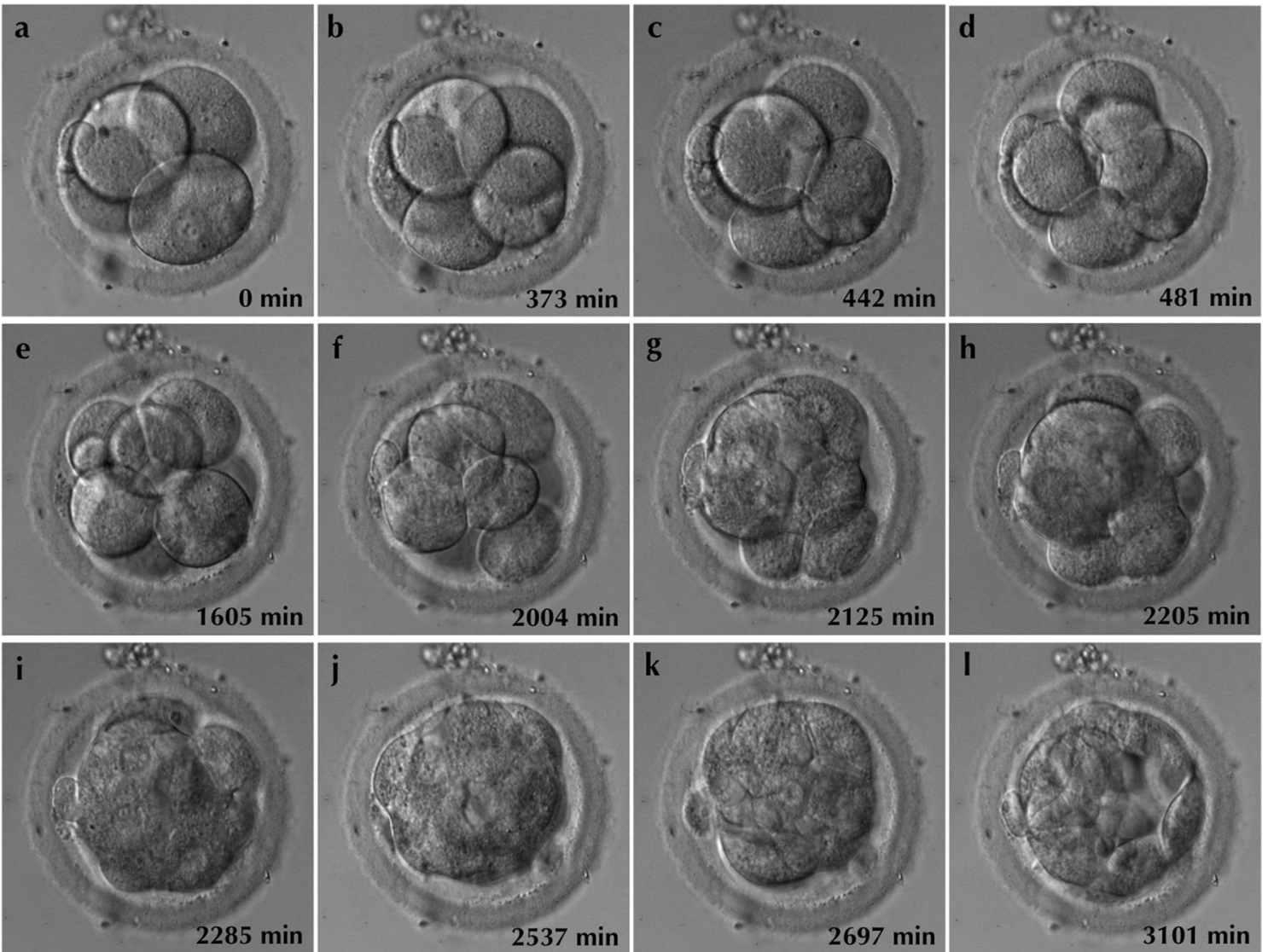

Fig. 2 Compaction in the human embryo. After several cell divisions (ae), the blastomeres became flattened (f), and the intercellular boundaries became obscured $(\mathbf{g}-\mathbf{i})$, until they finally unified in one cluster $(\mathbf{j}, \mathbf{k})$.

These morphological changes are called "compaction", and blastulation occurs only after complete compaction of the embryo (I)

\section{Statistical analysis}

\section{Results}

As expected from previous study [2, 9, 16, 17], TLC analysis of 115 embryos showed that the blastomeres became more

Fig. 3 Initiation of compaction in human embryos. The values above the histogram indicate the numbers of embryos that have commenced compaction at each cell stage. For the 115 embryos examined, the initiation of compaction was distributed from the 4-cell stage to the 16-cell stage, and $86.1 \%(99 / 115)$ started compaction at or just after the 8cell stage. In contrast, only $13.9 \%$ $(16 / 115)$ of the embryos started compaction before the 8 -cell stage

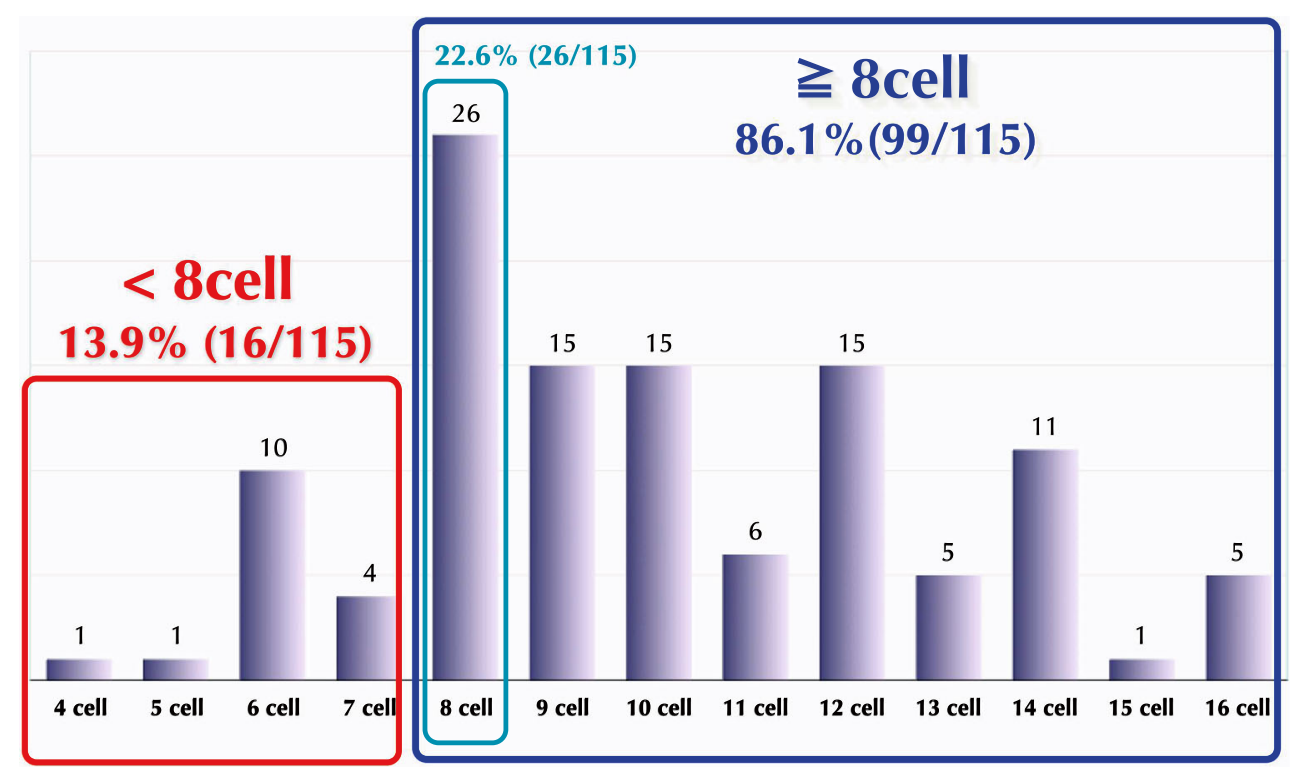


flattened, and the intercellular boundaries became obscured during compaction. Blastulation occurred after complete compaction of the embryos (Fig. 2, Supplemental Movie No. 1 (online resource 1)).

Although the initiation of compaction occurred from the 4cell to the 16-cell stage (Fig. 3), compaction most commonly commenced at the 8-cell stage (26/115 embryos; $22.6 \%)$. Furthermore, $86.1 \%(99 / 115)$ embryos initiated compaction at the 8-cell stage or later. Of these, $49.5 \%$ (49/99) developed into good quality blastocysts. In contrast, only $13.9 \%$ $(16 / 115)$ of embryos initiated compaction before the 8cell stage (Fig. 3), and only $18.8 \%(3 / 16)$ of these developed into good quality blastocysts (Table 1). Thus, good quality blastocysts were significantly more likely to develop from embryos that initiated compaction at the 8-cell stage or later, compared with embryos that initiated compaction earlier $(P=0.293$; Table 1$)$.

Moreover, $93.8 \%(15 / 16)$ of embryos that initiated compaction before the 8-cell stage showed multinucleation within at least one blastomere (Table 2). In addition, $62.5 \%(10 / 16)$ of the embryos that exhibited early initiation of compaction showed cytokinetic failure after formation of the cleavage furrow (Table 2, Fig. 4, Supplemental Movie No. 2 (online resource 2)).

\section{Discussion}

After several cellular divisions in the initial stages of embryonic development, the intercellular boundaries become obscured in a process called compaction, which maximizes intercellular contact and results in the formation of the morula. The cell-cell adhesion protein E-cadherin is first expressed during compaction, enabling the cells to adhere more tightly $[2,9,16-18]$. The obvious morphological changes are accompanied by functional changes resulting from the shift in the gene transcription profile from the maternal to the zygotic genome. These functional changes lead to the differentiation of the cells into the inner cell mass and trophectoderm, which is essential for blastocyst formation.

Here, by using our TLC technique, we observed a novel phenomenon regarding compaction in human embryonic development. Although the timing of the initiation of
Table 2 Incidence of MNBs and cytokinetic failure in early initiation of compaction $^{\mathrm{a}}$

\begin{tabular}{lll}
\hline $\begin{array}{l}\text { Compaction } \\
\text { stage }\end{array}$ & Incidence of MNBs $^{\mathrm{b}}$ & Incidence of cytokinetic failure \\
\hline 4 -cell & $100 \%(1 / 1)$ & $100 \%(1 / 1)$ \\
5 -cell & $100 \%(1 / 1)$ & $0 \%(0 / 1)$ \\
6 -cell & $100 \%(10 / 10)$ & $50 \%(5 / 10)$ \\
7 -cell & $75 \%(3 / 4)$ & $100 \%(4 / 4)$ \\
Total & $93.8 \%(15 / 16)$ & $62.5 \%(10 / 16)$ \\
\hline
\end{tabular}

${ }^{a}$ Of the 115 embryos analyzed, $16(13.9 \%)$ started compaction before the 8-cell stage

${ }^{\mathrm{b}}$ Proportion of embryos with at least one multinucleated blastomere (MNB)

compaction ranged from the 4-cell to 16-cell stage in human embryos, compaction was initiated at or just after the 8-cell stage in nearly $90 \%$ of the embryos analyzed. This result indicates that the initiation of compaction is physiologically likely to occur at or after the third mitotic division. In addition, while $49.5 \%$ of the embryos that initiated compaction after the 8-cell stage developed into good quality blastocysts, only $18.8 \%$ of the embryos that compacted earlier became good quality blastocysts. Furthermore, the embryos that underwent early initiation of compaction displayed a high incidence of MNBs (average, 93.8\%), and embryos with at least one MNB did not develop into good quality or hatched blastocysts. These data indicate that the early initiation of compaction is strongly associated with the incidence of MNBs, which in turn is related to polyploidy of the blastomeres. Moreover, $68.8 \%$ of the early-compacting embryos displayed cytokinetic failure after the cytoplasmic furrow had formed, indicating that the embryos with MNBs were generated from a novel mechanism whereby the blastomeres underwent cytokinetic failure with karyokinesis during mitotic division. Therefore, embryos with MNBs appear to have low developmental potential due to the presence of abnormal blastomeres (i.e., aberrant ploidy) [19].

Based on the incidence of MNBs and theoretical considerations, we hypothesize that embryos that initiate compaction at the 4-cell stage have cytokinetic failure with karyokinesis in all four blastomeres, embryos that initiate compaction at the 5cell stage are derived from one blastomere that cleaved

Table 1 Compaction initiation and developmental potential

\begin{tabular}{llll}
\hline Timing of compaction initiation & $<8$ cell $(n=16)$ & $\geq 8$ cell $(\mathrm{n}=99)$ & $P$-value \\
\hline Good quality blastocysts $^{\mathrm{a}}$ & $18.8 \%(3 / 16)$ & $49.5 \%(49 / 99)$ & $<0.05$ \\
Poor quality blastocysts & $81.2 \%(13 / 16)$ & $50.5 \%(50 / 99)$ & $<0.05$ \\
\hline
\end{tabular}

${ }^{\mathrm{a}}$ Good quality blastocysts are defined as being quality 3BB or greater (Gardner grading system) 

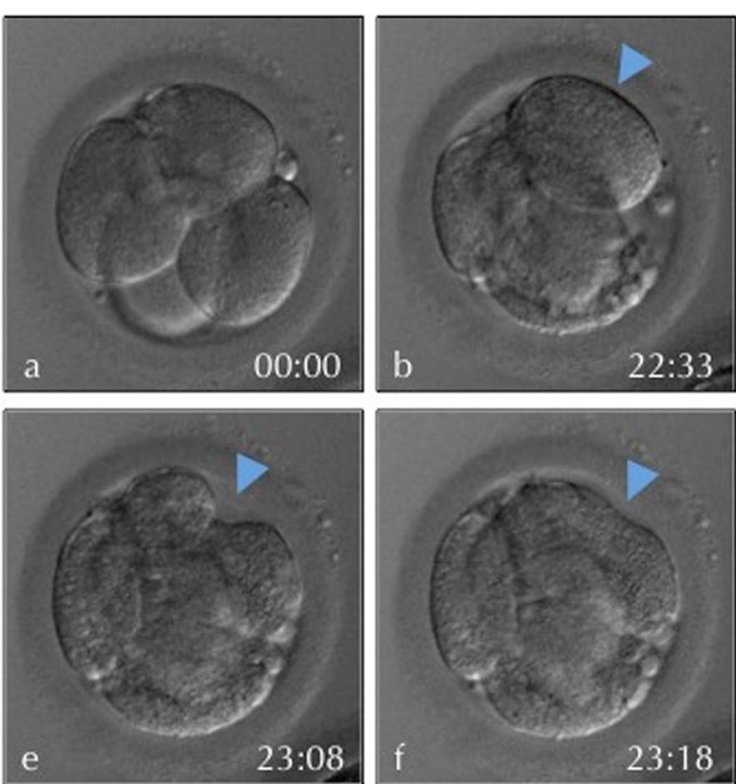

Fig. 4 Cytokinetic failure and multinucleated blastomere formation. Still-frames of a representative embryo showing the process of cytokinetic failure and multinucleated blastomere (MNB) formation are shown. In each panel, the time from the start of imaging is displayed (hours: minutes). At the 4-cell stage, the embryo showed one nucleus in each
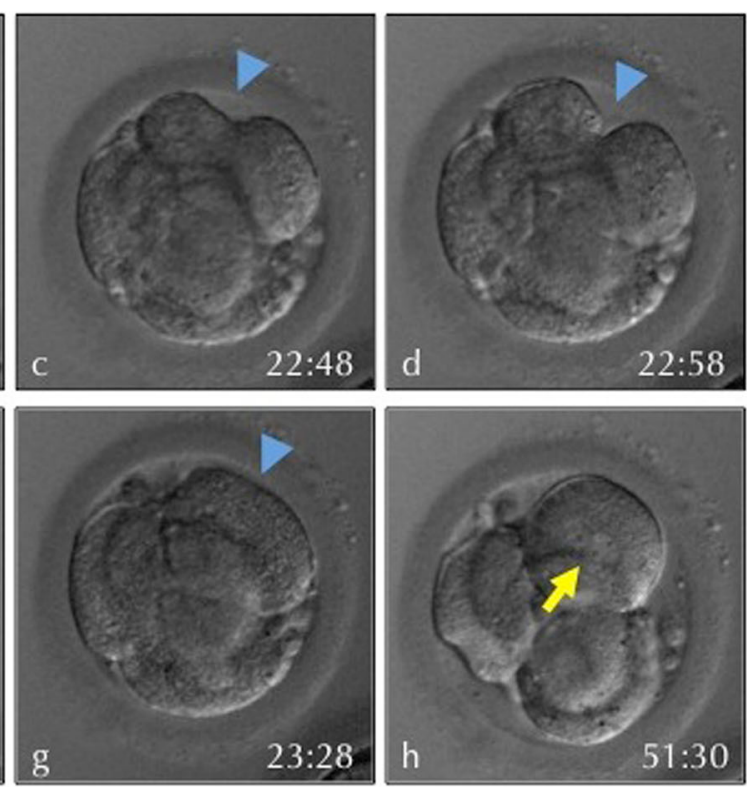

blastomere (a). At about $22 \mathrm{~h}$ into the time-lapse recording (b), one of the blastomeres started to cleave, and gradually formed the cleavage furrow (blue arrow; c, d), but then failed to cleave (e-g). At $51 \mathrm{~h}$ into the recording, the blastomere returned to its original shape, but with multinucleation (yellow arrow; $\mathbf{h}$ )

presence of MNBs is common in embryos that undergo early compaction indicates that the use of such embryos in clinical practice should be carefully considered.

Acknowledgments We would like to express our particular thanks to D. Payne and S. Flaherty in Adelaide, Australia, for their invaluable support in the development of a new in vitro culture system for timelapse cinematography. We also would like to especially thank $\mathrm{N}$. Murakami for image editing. Finally, this work was supported by all the staff in the Reproductive Centre, Mio Fertility Clinic.

\section{References}

1. Hurst PR, Jefferies K, Eckstein P, Wheeler AG. An ultrastructural study of preimplantation uterine embryos of the rhesus monkey. J Anat. 1978;126:209-20.

2. Enders AC, Lantz KC, Schlafke S. The morula-blastocyst transition in two old world primates: the baboon and rhesus monkey. J Med Primatol. 1990;19:725-47.

3. Alikani M, Calderon G, Tomkin G, Garrisi J, Kokot M, Cohen J. Cleavage anomalies in early human embryos and survival after prolonged culture in-vitro. Hum Reprod. 2000;15:2634-43.

4. Fleming TP, Hay M, Javed Q, Citi S. Localization of tight junction protein cingulin is temporally and spatially regulated during early mouse development. Development. 1993;117:1135-44.

5. Koyama H, Suzuki M, Yang X, Jiang S. FooteRH. Analysis of polarity of bovine and rabbit embryos by scanning electron microscopy. Biol Reprod. 1994;50:163-70.

6. Pratt HPM, Ziomek CA, Reeve WJD, Johnson MH. Compaction of the mouse embryo: an analysis of its components. J Embryol Exp Morph. 1982;270:113-32. the embryos had been frozen. However, our finding that the 
7. Ducibella T, Albertini DF, Anderson E, Biggers JD. The preimplantation mouse embryo: characterization of intercellular junctions and their appearance during development. Dev Biol. 1975;45:231-50.

8. Reeve WJD. Cytoplasmic polarity develops at compaction in rat and mouse embryos. J Embryol Exp Morphol. 1981;62:351-67.

9. Nikas G, Ao A, Winston RML, Handyside AH. Compaction and surface polarity in the human embryo in vitro. Biol Reprod. 1996;55: $32-7$.

10. Payne D, Flaherty SP, Barry MF, Matthews CD. Preliminary observations on polar body extrusion and pronuclear formation in human oocytes using time-lapse video cinematography. Hum Reprod. 1997;12:532-41.

11. Adachi Y, Takeshita C, Wakatsuki Y, Iwata K, Kato Y, Ueno Y, et al. Dynamic analysis of human embryos using time-lapse cinematography. J Mamm Ova Res. 2005;22:64-70.

12. Mio Y, Maeda K. Time-lapse cinematography of dynamic changes occurring during in vitro development of human embryos. Am J Obstet Gynecol. 2008;199:660e1-5.

13. Mio Y, Iwata K, Yumoto K, Kai Y, Sargant HC, Mizoguchi C, et al. Possible mechanism of polyspermy block in human oocytes observed by time-lapse cinematography. J Assist Reprod Genet. 2012;29:951-6.
14. Veeck LL. Preembryo grading and degree of cytoplasmic fragmentation (1999). In: an atlas of human gametes and conceptuses: an illustrated reference for assisted reproductive technology. New York, USA: Parthenon Publishing, 46-51

15. Gardner DK, Schoolcraft WB. In-vitro culture of human blastocysts. In: Jansen R, Mortimer D, editors. Towards reproductive certainty: fertility and genetics beyond 1999. Carnforth: Parthenon Press; 1999. p. $378-88$.

16. Fleming TP, Sheth B, Fesenko I. Cell adhesion in the preimplantation mammalian embryo and its role in trophectoderm differentiation and blastocyst morphogenesis. Front Biosci. 2001;6:D1000-1007.

17. Larue L, Ohsugi M, Hirchenhain J, Klemler R. E-cadherin null mutant embryos fail to form a trophectoderm epithelium. Proc Natl Acad Sci U S A. 1994;91:8263-7.

18. Bell CE, Calder MD, Watson AJ. Genomic RNA profiling and the programme controlling preimplantation mammalian development. Mol Hum Reprod. 2008;14:691-701.

19. Hardy K, Winston RML, Handyside AH. Binucleate blastomeres in preimplantation human embryos in vitro: failure of cytokinesis during early cleavage. J Reprod Fertil. 1993;98:549-58. 